\title{
Evaluation of lower limb kinetics during gait, sprint and hop tests before and after anterior cruciate ligament reconstruction
}

\author{
Joaquín Moya-Angeler $^{1,4}{ }_{(\mathbb{C})} \cdot$ Javier $_{\text {Vaquero }}{ }^{2}$ Francisco $_{\text {Forriol }}^{3}$
}

Received: 26 October 2015/Accepted: 13 March 2017/Published online: 30 March 2017

(c) The Author(s) 2017. This article is an open access publication

\begin{abstract}
Background The purpose of this study was to evaluate the functional status prior to and at different times after anterior cruciate ligament reconstruction (ACLR), and to analyze the changes in the kinetic patterns of the involved and uninvolved lower limb during gait, sprint and three hop tests. Materials and methods Seventy-four male patients with an ACL injury were included in the study. All patients performed a standardized kinetic protocol including gait, sprint and three hop tests (single-leg hop, drop vertical jump and vertical jump tests), preoperatively and at 3, 6, and 12 months after ACLR with a semitendinosus gracilis tendon autograft. Measurements were performed with two force plates. The lower limb symmetry index (LSI) was calculated to determine whether a side-to-side leg difference was classified as normal (LSI $>90 \%$ ) or abnormal (LSI $<90 \%$ ). Results The LSI presented high values $(>90 \%)$ at almost all times before and after ACLR in gait, sprint and singleleg hop tests $(p<0.005)$, with a tendency to increase postoperatively. A lower LSI was observed $(<90 \%)$ in tests
\end{abstract}

This work was carried out at the Hospital FREMAP Majadahonda in Madrid.

Joaquín Moya-Angeler

jmoyaangeler@gmail.com

1 Hospital Special Surgery, 535 E 70th St, New York City, NY 10021, USA

2 University Hospital Gregorio Marañon, Calle del Dr. Esquerdo, 46, 28007 Madrid, Spain

3 School of Medicine, University San Pablo-CEU, Madrid, Urbanización Montepríncipe, Boadilla del Monte, 28668 Madrid, Spain

$4 \quad 144$ E 74th St 3r, New York, NY 10021, USA where both extremities were tested simultaneously, such as the drop vertical jump and vertical hop tests $(p<0.05)$. Conclusion We observed a tendency to increase symmetry restoration in the kinetics of the involved and uninvolved limb up to twelve months after ACLR, especially in those tests, in which, both limbs were tested individually (gait analysis, sprint and single-leg hop tests). Therefore, the isolation of the involved and uninvolved limb seems to be a critical component in the functional rehabilitation and evaluation of patients before and after ACLR.

Level of evidence level III.

Keywords Knee kinetics · ACL deficiency - ACL reconstruction $\cdot$ Hop tests $\cdot$ Semitendinosus gracilis autograft

\section{Introduction}

Anterior cruciate ligament (ACL) injuries commonly lead to abnormal kinematics, kinetics, and muscle activity of the injured extremity. For that reason, it has been suggested that knee function should be examined and considered in the decision making process for ACL reconstruction (ACLR) $[1,2]$. Including functional assessments in the evaluation of patients after ACL injury increases our ability to decide who should later undergo ACLR (non-copers) and who may benefit from non-operative management (copers) [3]. These performance-based measures are also important indicators of knee function after ACLR $[4,5]$.

Knee instability in ACL-deficient individuals has traditionally been assessed using static measures; however, knee instability during dynamic activities is not related to passive measures [6]. Different gait adaptations have been observed in non-copers (individuals who experience knee 
instability after ACL rupture) soon after ACL injury, which seem to be consistent with their movement and muscle activity during jogging [6-8]. Hop tests are performancebased measures used to assess the combination of muscle strength, neuromuscular control, confidence in the limb, and the ability to tolerate loads related to sports-specific activities [9-12]. These tests can detect limb asymmetries in patients before and after ACLR. However, while unilateral deficits are present in patients after ACLR, these may not be evident during activities involving both lower extremities. For this reason, it has been suggested that isolation of the involved limb with unilateral hop tests should be performed to detect discrepancies in function [13]. Previous studies have shown symmetry restoration and functional recovery before and after ACLR after evaluating the hop distances and times of the involved and uninvolved extremity $[14,15]$. However, to our knowledge, this is the first study evaluating the kinetics of the injured and non-injured limb (before and after ACLR) during different strenuous activities, ranging from simple walking (gait analysis) to sprint, and different hop tests (including single-leg and bilateral tests).

The purpose of this study was, therefore, to evaluate the functional status prior to and at different times after ACLR, and to analyze the changes in the kinetic patterns of the involved and uninvolved limb lower during gait, sprint and three hop tests.

\section{Materials and methods}

Between January 2007 and May 2009, 105 patients with unilateral ACL injury were recruited for this study. Inclusion criteria were males aged between 20 and 40 years, with a documented and symptomatic ACL injury associated or not to a meniscal tear sustained within the previous three months. Patients were excluded if they presented any concomitant musculoskeletal condition or previous intervention in the lower extremities that could alter the mechanics of the limb (Table 1). All patients were physically active and were able to perform regular daily activities. Before undergoing ACLR, all patients performed a 6-week progressive exercise training program, emphasizing aggressive quadriceps strengthening to restore muscle strength, range of motion and appropriate neuromuscular responses [16].

After concluding this rehabilitation program, all patients completed a standard kinetic protocol which was performed the day before the operation. All patients underwent primary unilateral ACLR using a semitendinosus gracilis tendon autograft obtained from the ipsilateral leg. After surgery, all participants followed the same rehabilitation guidelines [16], and they repeated the same kinetic protocol at 3, 6 and 12 months after the operation. Following surgery, all subjects exhibited full range of motion
Table 1 Patient demographics

\begin{tabular}{ll}
\hline Initial study sample & 105 patients \\
Cartilage lesions & 23 patients \\
Posterior cruciate ligament injury & 1 patients \\
Meniscal tears & 5 patients \\
Medial collateral ligament/lateral & 2 patients \\
$\quad$ collateral ligament injury & \\
Final study sample & 74 patients \\
Lost to follow-up & 3 patients \\
Age (years) & $34.0(\mathrm{SD}=9)$ \\
Mean weight (Newton) & $843.0(\mathrm{SD}=20.32)$ \\
Right knees & $46(62 \%)$ \\
Left knees & $28(38 \%)$ \\
Mean follow-up & 12 months \\
\hline
\end{tabular}

A

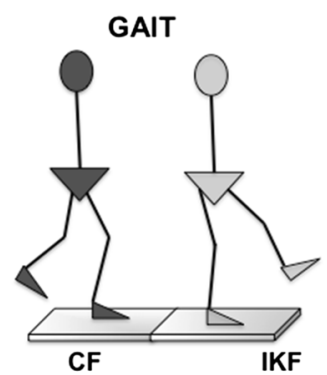

Heel MVF (N) Impulse MVF (N)

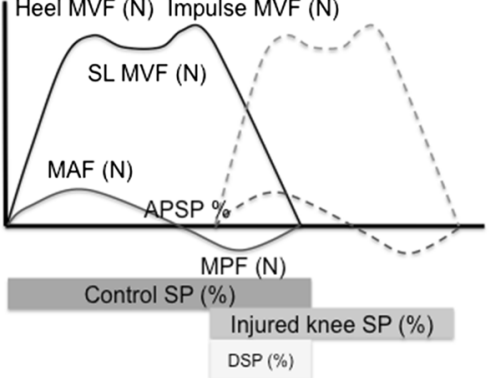

B Sprint

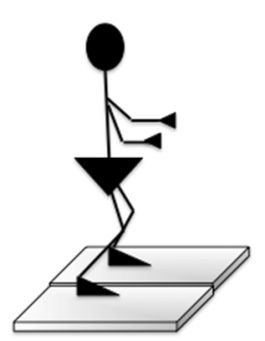

Maximum Vertical Force

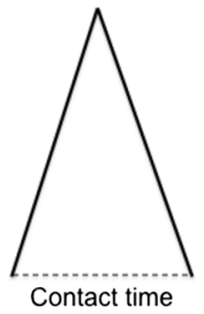

Fig. 1 a Gait test and kinetics graph. Subjects walked along a 5-m wooden walkway in which one of the force plates was embedded. Subjects were told to walk at a self-selected comfortable pace. b Sprint test and kinetics graph. The sprint test was performed with the patient standing on both platforms. After an initial trial, they were instructed to sprint as fast as possible for $5 \mathrm{~s} \mathrm{(CF} \mathrm{control} \mathrm{foot,} \mathrm{IKF}$ injured-knee foot, $M a x$ maximum, $A P$ anterior-posterior)

of the knee, none to minimal joint effusion, and none to minimal pain during ambulation. None of the patients reported episodes of the knee 'giving way'.

The kinetic protocol included gait analysis, sprint and hop tests (single-leg hop test, drop vertical jump and vertical hop test) (Figs. 1, 2, 3, 4) [17]. All measurements were performed with the use of two Kistler force plates (Kistler $^{\circledR}$; Winterthur, Switzerland) measuring $60 \times 90 \mathrm{~cm}$, fixed onto the floor in front of each other. Parameters obtained during gait for the control foot (CF) and injured knee-foot (IKF) included (Fig. 1a) step 

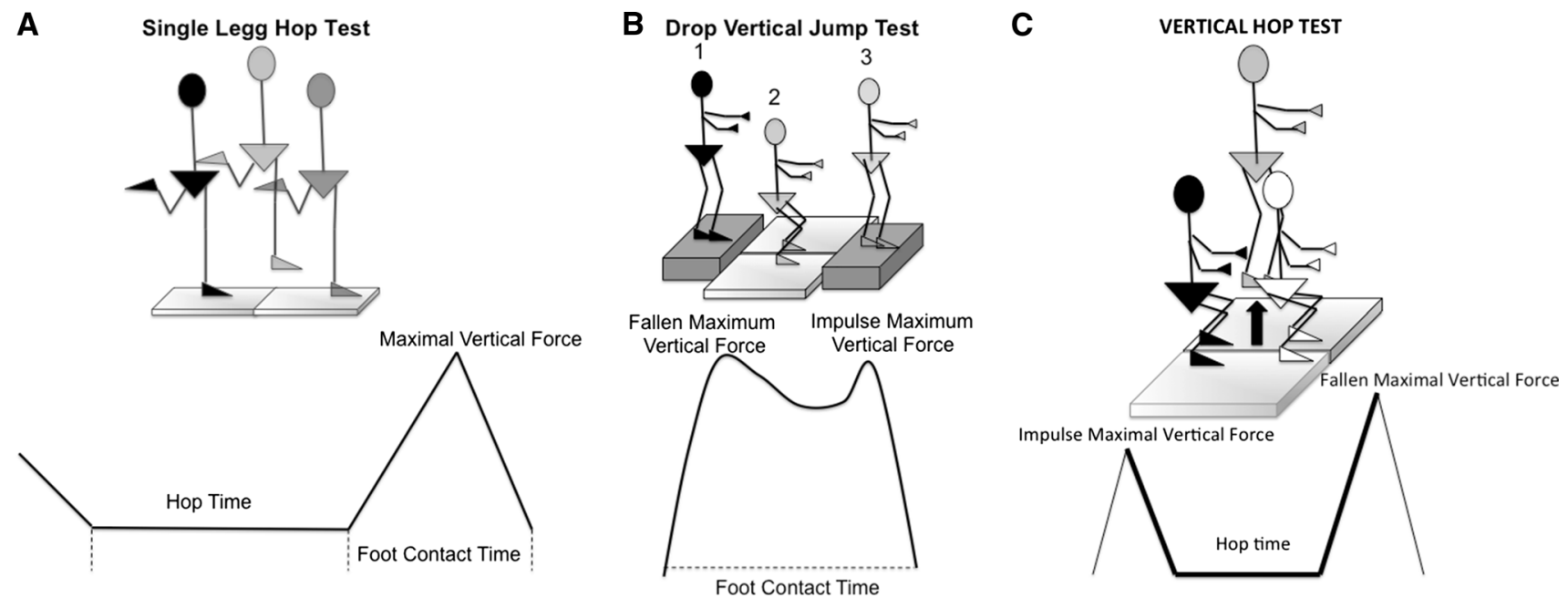

Fig. 2 a Single-leg hop test and kinetics graph. The single-leg hop test for distance was performed as previously described [18]. Patients were instructed to stand on one leg and to position their toes against a mark on the floor. They were then instructed to hop forward as far as possible and to land on the same leg. b Drop vertical jump and kinetics graph. Subjects were instructed to drop off a 30-cm box and

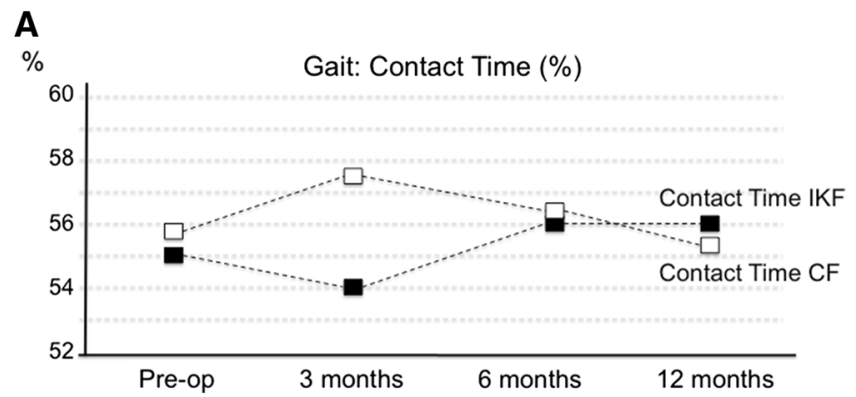

C

Force / Gait: Anterior and Posterior Maximum Vertical Forces Weight \%

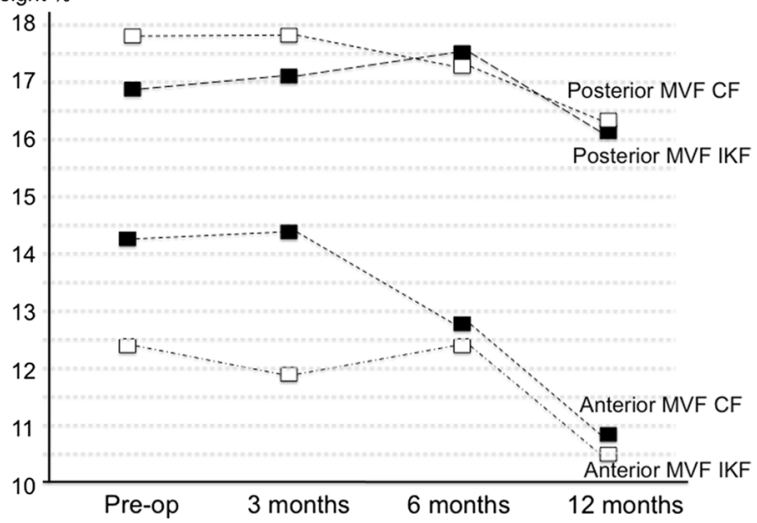

perform a maximum jump after landing. The box distance was adjusted so that the patient could land with one foot on each platform. c Vertical hop test and kinetics graph. Vertical hop test was performed (Fig. 2c) with the patient standing on both platforms and being instructed to hop using his arms as countermovement. Max maximum

\section{B}
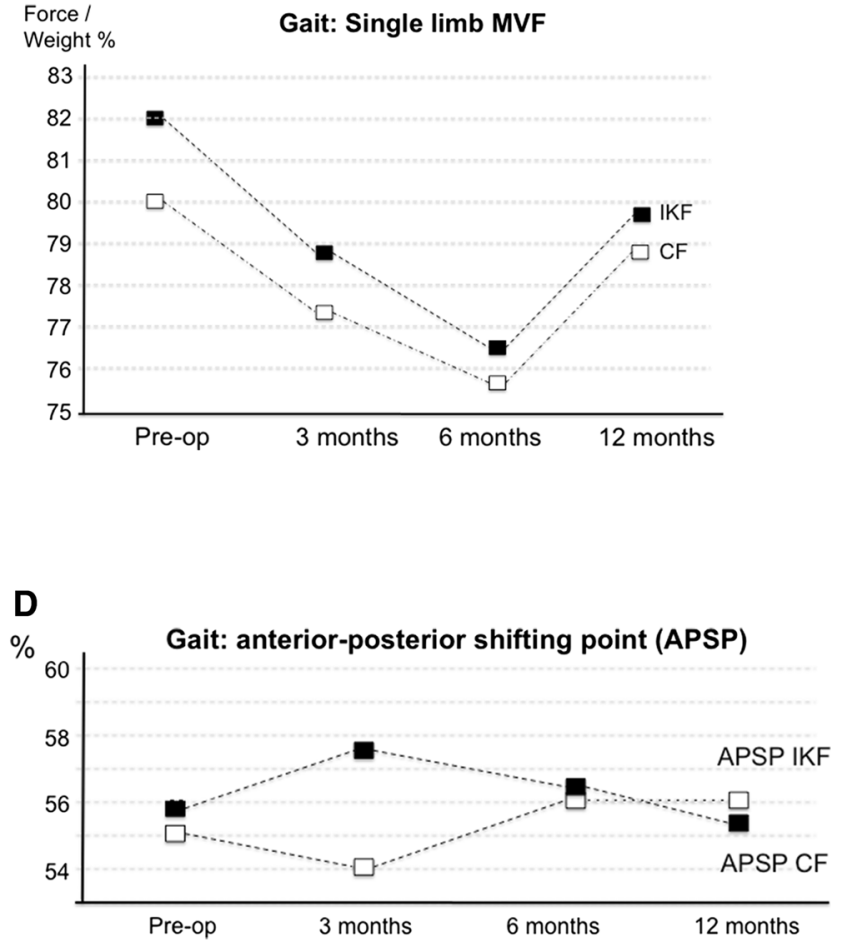

Fig. 3 Gait kinetics, a contact time, b single-leg stance vertical force, $\mathbf{c}$ maximum anterior and posterior forces, $\mathbf{d}$ anterior posterior shifting point. $I K N$ injured-knee foot, $C F$ control foot, Pre-op preoperatively

percentage (SP), double-limb step percentage (DSP), anterior-posterior shifting point (APSP) contact time (CT), heel maximum vertical force (MVF), single-limb (SL)
MVF, impulse MVF, maximum anterior force (MAF) and maximum posterior force (MPF). Sprint test parameters included (Fig. 1b) MVF and CT. Parameters obtained from 


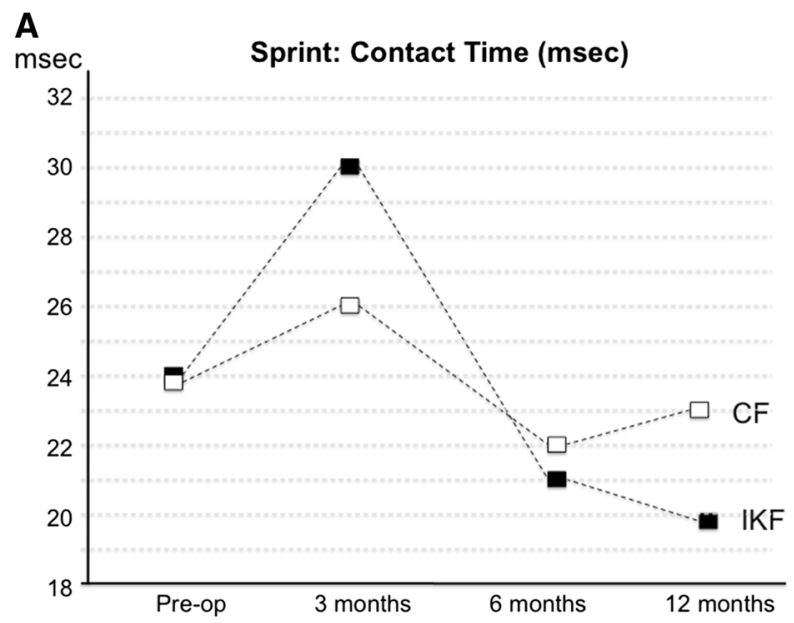

C

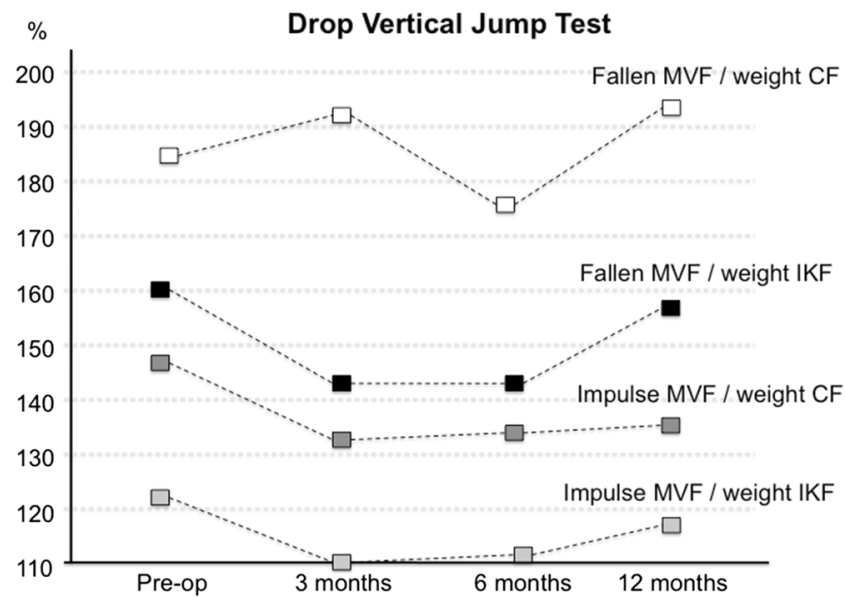

B

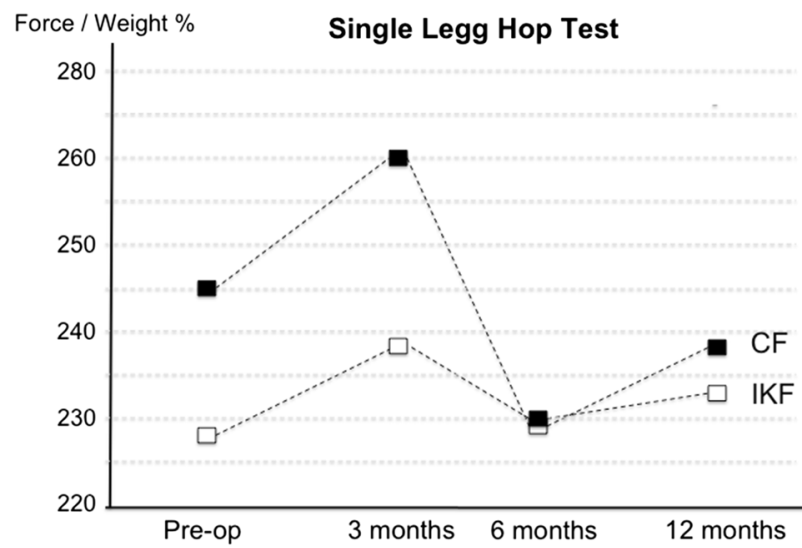

D

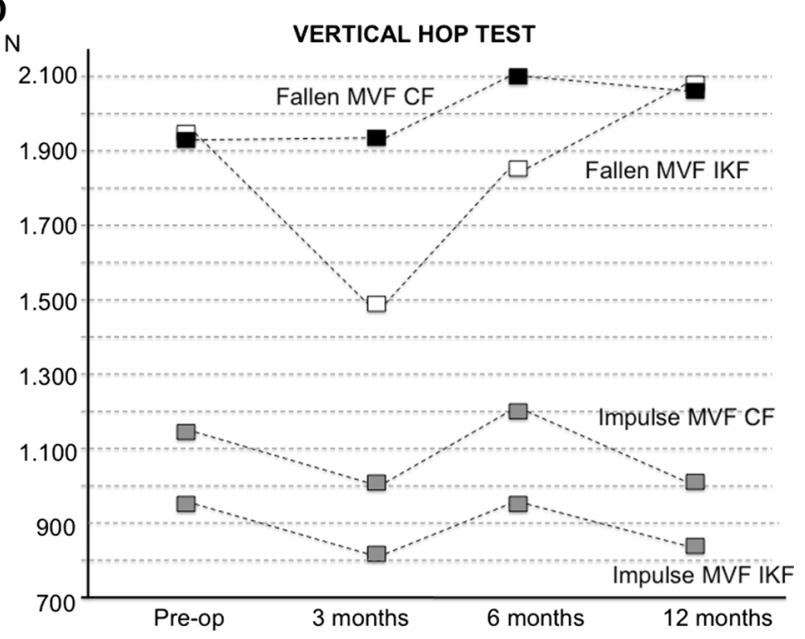

Fig. 4 a Sprint kinetics, b single-leg hop test kinetics, c drop vertical jump kinetics, d vertical hop test kinetics. IKF injured-knee foot, $C F$ control foot, Pre-op preoperatively

the single-leg hop test included (Fig. 2a) hop time, MVF and CT. Drop vertical jump parameters included (Fig. 2b) fallen MVF, CT and impulse MVF. Vertical hop test parameters included (Fig. 2c) impulse MVF, hop time and fallen MVF. All parameters were normalized by body weight. The lower limb symmetry index (LSI) was calculated to determine whether a side-to-side leg difference was classified as normal $(>90 \%)$ or abnormal $(<90 \%)$ [18]. The LSI was defined as the ratio of the involved limb score and the uninvolved limb score expressed in percentage (involved/uninvolved $\times 100=\mathrm{LSI}$ ). Although LSI scores were the outcome measures of most interest, absolute scores on each lower extremity were also presented for a better understanding of the calculated index score (Tables 2, 3, 4, 5, 6). Patients were carefully instructed on how to conduct each trial before the definitive test was performed (Figs. 1, 2). Data were reviewed for completeness after each trial, and data collection continued until a minimum of three trials were recorded for both limbs. The hop tests were considered valid if the landing was stable.
The timing of the kinetic profiles was normalized as a percentage of a single complete cycle.

Descriptive statistics, including mean and standard deviation, were used to describe patient demographics. Mean kinetic values at baseline and at 3, 6, and 12 months postoperatively were compared using repeated-measures analyses of variance (ANOVA). For each ANOVA in a significant $\mathrm{F}$ ratio, post hoc analysis was performed using $t$ test with Bonferroni correction for multiple comparisons; this was performed in order to look at the individual effect rather than the effect of all variables together. All statistical analyses were performed using SPSS v.17.0 for Windows (Chicago, IL, USA). Statistical significance was set as $p<0.05$.

\section{Results}

Results of gait kinetics are shown in Table 2 (Figs. 1, 2). Although the LSI improved 12 months after surgery for most of the measurements performed, these differences 
Table 2 Gait kinetics

\begin{tabular}{|c|c|c|c|c|c|c|c|c|}
\hline & \multicolumn{2}{|c|}{ Preoperative } & \multicolumn{2}{|c|}{3 months } & \multicolumn{2}{|c|}{6 months } & \multicolumn{2}{|c|}{12 months } \\
\hline & $X$ & SD & $X$ & $\mathrm{SD}$ & $X$ & $\mathrm{SD}$ & $X$ & $\mathrm{SD}$ \\
\hline \multicolumn{9}{|c|}{ MVF heel } \\
\hline IKF & 95.51 & 1.01 & 93.39 & 2.70 & 92.27 & 2.58 & 92.88 & 2.71 \\
\hline $\mathrm{CF}$ & 99.81 & 1.58 & 98.93 & 2.19 & 94.88 & 3.15 & 95.46 & 2.2 \\
\hline$p$ & 0.001 & & 0.061 & & 0.512 & & 0.312 & \\
\hline LSI & $95.6 \%$ & & $94.4 \%$ & & $97.2 \%$ & & $97.2 \%$ & \\
\hline \multicolumn{9}{|c|}{ Single-leg MVF } \\
\hline $\mathrm{IKF}$ & 82.17 & 1.3 & 78.94 & 2.86 & 76.47 & 2.75 & 79.92 & 1.5 \\
\hline $\mathrm{CF}$ & 80.15 & 1.28 & 77.23 & 2.81 & 75.88 & 1.68 & 78.99 & $2.3^{3}$ \\
\hline$p$ & 0.001 & & 0.050 & & 0.0561 & & 0.061 & \\
\hline LSI & $102 \%$ & & $102 \%$ & & $100 \%$ & & $101 \%$ & \\
\hline \multicolumn{9}{|c|}{$\operatorname{IVF}(\%)$} \\
\hline IKF & 96.93 & 1.57 & 95.49 & 3.18 & 93.34 & 2.31 & 94.69 & 2.66 \\
\hline $\mathrm{CF}$ & 100.39 & 1.58 & 95.81 & 2.76 & 90.13 & 4.97 & 93.69 & 3.37 \\
\hline$p$ & 0.001 & & 0.222 & & 0.061 & & 0.712 & \\
\hline LSI & $96 \%$ & & $99.6 \%$ & & $103 \%$ & & $101 \%$ & \\
\hline \multicolumn{9}{|c|}{ Anterior force } \\
\hline IKF & 12.39 & 4.25 & 11.58 & 5.31 & 12.34 & 5.7 & 10.54 & 4.57 \\
\hline $\mathrm{CF}$ & 14.36 & 1.58 & 14.42 & 3.61 & 12.78 & 4.43 & 10.80 & 4.10 \\
\hline$p$ & 0.001 & & 0.069 & & 0.332 & & 0.077 & \\
\hline LSI & $86.2 \%$ & & $80.3 \%$ & & $96.5 \%$ & & $97.5 \%$ & \\
\hline \multicolumn{9}{|c|}{ Posterior force } \\
\hline IKF & 16.95 & 0.63 & 17.06 & 1.30 & 17.51 & 0.84 & 16.05 & 0.6 \\
\hline $\mathrm{CF}$ & 17.71 & 0.52 & 17.75 & 0.95 & 17.15 & 0.97 & 16.29 & 1.21 \\
\hline$p$ & 0.01 & & 0.073 & & 0.0912 & & 0.057 & \\
\hline LSI & $95.7 \%$ & & $96.1 \%$ & & $102 \%$ & & $98.5 \%$ & \\
\hline \multicolumn{9}{|c|}{ Contact time $(\%)$} \\
\hline IKF & 55.72 & 0.45 & 54.29 & 0.69 & 56.74 & 1.66 & 56.24 & 0.96 \\
\hline $\mathrm{CF}$ & 55.85 & 0.39 & 57.50 & 0.88 & 56.14 & 1.05 & 55.30 & 0.53 \\
\hline$p$ & 0.069 & & 0.0012 & & 0.067 & & 0.078 & \\
\hline LSI & $99.7 \%$ & & $94.4 \%$ & & $101 \%$ & & $101 \%$ & \\
\hline
\end{tabular}

$M V F$ maximum vertical force, $I V F$ impulse vertical force, $A P$ anterior-posterior, $I K F$ injured-knee foot, $C F$ control foot, $S D$ standard deviation

were not statistically significant. The only significant difference was the preoperative and 12-month anterior force; however, this difference was not statistically significant ( $p$ 0.077). Contact times showed no differences pre- and postoperatively. The sprint kinetics results (Table 3) presented a similar pattern; however, a slight improvement in LSI was observed 12 months after ACLR ( $p$ 0.078). Single-leg hop test kinetics (Table 4; Fig. 4b) presented a significant improvement in LSI 6 months (100\%) after ACLR, which persisted up to 12 months postoperatively (98.2\%) ( $p$ 0.001-0.015). However, drop vertical jump results (Table 5, Fig. 4c) presented a different pattern with a lower LSI 12 months after surgery $(p 0.002)(<90 \%$ at all
Table 3 Sprint kinetics

\begin{tabular}{|c|c|c|c|c|c|c|c|c|}
\hline & \multicolumn{2}{|c|}{ Preoperative } & \multicolumn{2}{|c|}{3 months } & \multicolumn{2}{|c|}{6 months } & \multicolumn{2}{|c|}{12 months } \\
\hline & $X$ & SD & $X$ & SD & $X$ & SD & $X$ & $\mathrm{SD}$ \\
\hline \multicolumn{9}{|l|}{ MVF } \\
\hline IKF & 180.5 & 38.4 & 157.4 & 37.5 & 168.6 & 41.4 & 180.6 & 31.4 \\
\hline $\mathrm{CF}$ & 190.2 & 32.7 & 193.8 & 27.5 & 183.9 & 24.0 & 184.1 & 29.9 \\
\hline$p$ & 0.052 & & 0.521 & & 0.067 & & 0.078 & \\
\hline LSI & $94.9 \%$ & & $81.2 \%$ & & $91.6 \%$ & & $98 \%$ & \\
\hline \multicolumn{9}{|c|}{ Contact time } \\
\hline IKF & 0.24 & 0.13 & 0.28 & 0.19 & 0.22 & 0.08 & 0.18 & 0.06 \\
\hline $\mathrm{CF}$ & 0.24 & 0.13 & 0.25 & 0.20 & 0.21 & 0.09 & 0.25 & 0.20 \\
\hline$p$ & 0.067 & & 0.172 & & 0.050 & & 0.101 & \\
\hline LSI & $100 \%$ & & $112 \%$ & & $104 \%$ & & $72 \%$ & \\
\hline
\end{tabular}

$M V F$ maximum vertical force, $I K F$ injured-knee foot, $C F$ control foot, $S D$ standard deviation

Table 4 Single-leg hop test kinetics

\begin{tabular}{|c|c|c|c|c|c|c|c|c|}
\hline & \multicolumn{2}{|c|}{ Preoperative } & \multicolumn{2}{|c|}{3 months } & \multicolumn{2}{|c|}{6 months } & \multicolumn{2}{|c|}{12 months } \\
\hline & $X$ & $\mathrm{SD}$ & $X$ & SD & $X$ & SD & $X$ & SD \\
\hline \multicolumn{9}{|l|}{ MVF } \\
\hline IKF & 228.4 & 66.9 & 238.8 & 42.7 & 230.6 & 42.4 & 233.6 & 26.85 \\
\hline $\mathrm{CF}$ & 245.7 & 45.1 & 260.6 & 46.6 & 229.6 & 42.8 & 237.8 & 58.56 \\
\hline$p$ & 0.035 & & 0.001 & & 0.001 & & 0.015 & \\
\hline LSI & $92.9 \%$ & & $91.6 \%$ & & $100 \%$ & & $98.2 \%$ & \\
\hline \multicolumn{9}{|c|}{ Hop time } \\
\hline IKF & 0.18 & 0.11 & 0.17 & 0.04 & 0.14 & 0.09 & 0.22 & 0.21 \\
\hline $\mathrm{CF}$ & 0.18 & 0.09 & 0.17 & 0.07 & 0.11 & 0.06 & 0.23 & 0.24 \\
\hline$p$ & 0.324 & & 0.823 & & 0.051 & & 0.823 & \\
\hline LSI & $100 \%$ & & $100 \%$ & & $127 \%$ & & $95 \%$ & \\
\hline \multicolumn{9}{|l|}{ CT } \\
\hline IKF & 0.44 & 0.19 & 0.38 & 0.1 & 0.38 & 0.1 & 0.37 & 0.11 \\
\hline $\mathrm{CF}$ & 0.38 & 0.14 & 0.39 & 0.14 & 0.37 & 0.13 & 0.38 & 0.09 \\
\hline$p$ & 0.005 & & 0.081 & & 0.143 & & 0.071 & \\
\hline LSI & $115 \%$ & & $97.4 \%$ & & $102.7 \%$ & & $97.3 \%$ & \\
\hline \multicolumn{9}{|c|}{ CT/hop time } \\
\hline IKF & 0.56 & 0.9 & 0.5 & 0.3 & 0.42 & 0.4 & 0.61 & 0.5 \\
\hline $\mathrm{CF}$ & 0.54 & 0.4 & 0.45 & 0.2 & 0.33 & 0.18 & 0.63 & 0.4 \\
\hline$p$ & 0.044 & & 0.051 & & 0.026 & & 0.007 & \\
\hline LSI & $103 \%$ & & $111 \%$ & & $127 \%$ & & $96.8 \%$ & \\
\hline
\end{tabular}

$M V F$ maximum vertical force, $I K F$ injured-knee foot, $C F$ control foot, $C T$ contact time, $S D$ standard deviation

times). Vertical hop test kinetics showed no differences between preoperative and postoperative LSI values $(<90 \%$ at all times) (Table 6). Contact/hop times showed no differences preoperatively or postoperatively in all test performed. 
Table 5 Drop vertical jump kinetics

\begin{tabular}{|c|c|c|c|c|c|c|c|c|}
\hline & \multicolumn{2}{|c|}{ Preoperative } & \multicolumn{2}{|c|}{3 months } & \multicolumn{2}{|c|}{6 months } & \multicolumn{2}{|c|}{12 months } \\
\hline & $X$ & SD & $X$ & SD & $X$ & SD & $X$ & SD \\
\hline \multicolumn{9}{|l|}{ FVF } \\
\hline IKF & 160.1 & 6.31 & 144.6 & 15.7 & 143.1 & 7.96 & 157.5 & 8.27 \\
\hline $\mathrm{CF}$ & 184.66 & 6.5 & 191.5 & 14.2 & 176.9 & 10.1 & 193.5 & 13.2 \\
\hline$p$ & 0.003 & & 0.001 & & 0.054 & & 0.002 & \\
\hline LSI & $86.6 \%$ & & $75 \%$ & & $80 \%$ & & $81.3 \%$ & \\
\hline \multicolumn{9}{|l|}{ IVF } \\
\hline IKF & 121.4 & 5.36 & 106.5 & 14.2 & 111.9 & 6.82 & 117.4 & 6.25 \\
\hline $\mathrm{CF}$ & 146.6 & 5.14 & 133.6 & 12.3 & 134.9 & 7.96 & 135.4 & 10.4 \\
\hline$p$ & 0.001 & & 0.043 & & 0.027 & & 0.007 & \\
\hline LSI & $82.8 \%$ & & $79.7 \%$ & & $82.9 \%$ & & $86.7 \%$ & \\
\hline \multicolumn{9}{|l|}{$\mathrm{CT}$} \\
\hline IKF & 0.63 & 0.06 & 0.46 & 0.05 & 0.53 & 0.05 & 0.64 & 0.14 \\
\hline $\mathrm{CF}$ & 0.62 & 0.05 & 0.5 & 0.05 & 0.52 & 0.05 & 0.65 & 0.13 \\
\hline$p$ & 0.567 & & 0.154 & & 0.061 & & 0.077 & \\
\hline LSI & $101 \%$ & & $92 \%$ & & $101 \%$ & & $98.4 \%$ & \\
\hline
\end{tabular}

$F V F$ fallen vertical force, $I V F$ impulse vertical force, $I K F$ injuredknee foot, $C F$ control foot, $S D$ standard deviation, $C T$ contact time

Table 6 Vertical hop test kinetics

\begin{tabular}{|c|c|c|c|c|c|c|c|c|}
\hline & \multicolumn{2}{|c|}{ Preoperative } & \multicolumn{2}{|c|}{3 months } & \multicolumn{2}{|c|}{6 months } & \multicolumn{2}{|c|}{12 months } \\
\hline & $X$ & SD & $X$ & SD & $X$ & SD & $X$ & SD \\
\hline \multicolumn{9}{|l|}{ IVF } \\
\hline IKF & 113.4 & 25.8 & 102.5 & 23.6 & 107.0 & 38.2 & 100.2 & 12.56 \\
\hline $\mathrm{CF}$ & 136.6 & 36.3 & 127.7 & 30.2 & 134.8 & 32.5 & 120.5 & 15.89 \\
\hline$p$ & 0.035 & & 0.001 & & 0.001 & & 0.015 & \\
\hline LSI & $83 \%$ & & $80 \%$ & & $79.3 \%$ & & $83.1 \%$ & \\
\hline \multicolumn{9}{|l|}{ FVF } \\
\hline IKF & 233.5 & 88.9 & 185.2 & 71.8 & 210.1 & 60.7 & 250.3 & 64.08 \\
\hline $\mathrm{CF}$ & 234.0 & 67.6 & 239.5 & 63.3 & 236.2 & 50.7 & 243.6 & 31.13 \\
\hline$p$ & 0.035 & & 0.080 & & 0.200 & & 0.063 & \\
\hline LSI & $99.7 \%$ & & $77.3 \%$ & & $88.9 \%$ & & $102 \%$ & \\
\hline \multicolumn{9}{|c|}{ IVF/FVF } \\
\hline IKF & 59.93 & 48.3 & 60.16 & 18.9 & 55.29 & 27.1 & 42.43 & 12.02 \\
\hline $\mathrm{CF}$ & 64.09 & 30.6 & 55.39 & 13.9 & 59.94 & 24.9 & 50.04 & 7.83 \\
\hline$p$ & 0.035 & & 0.432 & & 0.587 & & 0.156 & \\
\hline LSI & $93.5 \%$ & & $108.6 \%$ & & $92.2 \%$ & & $84.5 \%$ & \\
\hline \multicolumn{9}{|c|}{ Hop time } \\
\hline IKF & 0.42 & 0.11 & 0.46 & 0.11 & 0.48 & 0.07 & 0.47 & 0.04 \\
\hline $\mathrm{CF}$ & 0.41 & 0.11 & 0.53 & 0.22 & 0.47 & 0.07 & 0.39 & 0.14 \\
\hline$p$ & 0.057 & & 0.762 & & 0.052 & & 0.062 & \\
\hline LSI & $102 \%$ & & $86.7 \%$ & & $102 \%$ & & $120 \%$ & \\
\hline
\end{tabular}

$I V F$ impulse vertical force, $F V F$ fallen vertical force, $M V F$ maximum vertical force, $I K F$ injured-knee foot, $C F$ control foot, $S D$ standard deviation

\section{Discussion}

The most significant finding of this study is that limb to limb kinetic asymmetries presented a tendency to decrease with time after ACLR in the gait, sprint and single-leg hop tests, with the LSI $>90 \%$ before and after ACLR. The drop vertical jump and vertical hop tests, however, did not present such behavior with the LSI $<90 \%$ before and after ACLR.

Our results seem to be consistent with those reported by other authors $[14,15]$, showing symmetry restoration and functional recovery before and after ACLR in gait, sprint and single-leg hop tests. However, we were not able to observe this phenomenon in all tests performed, since both the drop vertical jump and the vertical hop test did not improve their LSI after ACLR. Logerstedt et al. [15] evaluated functional recovery (quadriceps strength testing, hop testing, and self-reported questionnaires for knee function) in eighty-three athletes after an ACL injury, and at different times after ACLR. They concluded that limb to limb asymmetries are reduced, and normal limb symmetry is returned to a similar level 6 months after ACLR. More recently, Rohman et al. [14] also evaluated changes in the involved and uninvolved limb function after ACLR in 122 patients, with twelve individual tests. From the twelve functional tests in the study, the single-leg squat, retro stepup, single-leg hop, crossover triple hop, and timed hop were suggested to be highly useful tests, since all showed an initial LSI $<90 \%$, with significant improvement after rehabilitation. To our knowledge, our study is the first to evaluate LSI functional kinetics in patients before and after ACLR. We included gait, sprint and different hop tests in order to find out if more demanding tests would show any differences. However, we observed that those tests in which the involved and uninvolved leg were tested individually (gait, spring and single-leg hop test) presented a high LSI (>90\%) before ACLR, with a tendency to increase at latest follow-up (close to 100\%). Nevertheless, those tests in which both legs were tested at the same time (drop vertical jump and vertical hop test) presented a low LSI preoperatively and at all times postoperatively.

Patient management after ACL injury in active individuals may be improved by evaluating function as a consequence of dynamic knee stability using simple hop tests and validated knee outcome surveys, rather than the magnitude of knee laxity and preinjury activity level $[19,20]$. Clinicians have traditionally used single-leg hop tests to assess both the patient's lower extremity muscular strength and the ability to perform tasks that challenge the stability of the knee [21, 22]. For that reason, single-leg hop tests are now commonly used in knee rehabilitation programs. Noyes et al. [23] were one of the first authors to 
describe a combination of hop tests that mimic the demands of dynamic knee stability during highly demanding activities, and are intended to prepare the patient for a return to such activities [24]. Posteriorly, Gustavsson et al. [25] reported high test-retest reliability, sensitivity, and accuracy after combining three hop tests, that included vertical jump, hop test for distance and hop test performance while developing fatigue (the side hop). More recently, single-leg hop tests have been used to detect persistent limb asymmetries in performance during highdemanding activities, using the lower symmetry index to evaluate the performance between the involved and uninvolved limb $[14,15]$. This is preferable to the use of singlelimb performance variables because both patients differ in ability, and because (in biomechanical testing) limb symmetry is associated with better rates of return to sports and lower rates of reinjury [24, 26]. Moreover, the current bibliography supports the use of LSI thresholds ranging from $80-90 \%$ before recommending return to sports $[24,27,28]$. Nevertheless, the effects of postoperative rehabilitation on the uninvolved limb are not well understood in regard to functional testing. It has been suggested that differences in postural stability after ACLR may be explained by the specific nature of the exercise, and by a possible compensation of the uninvolved lower extremity $[21,29,30]$. Therefore, while unilateral deficits are present in patients after ACLR, these may not be evident during activities involving both lower extremities. For this reason, it has been suggested that the isolation of the involved limb with unilateral hop tests should be performed to detect discrepancies in function [13]. This phenomenon which is not yet well understood, and presents inconclusive data in the literature, may explain the fact that in our study the LSI never improved in tests in which both the involved and uninvolved limb were tested at the same time.

This study presents some limitations. The results can only be generalized to subjects who present with isolated ACL injury, and should not be generalized to individuals with complex concomitant injuries. In addition, as the aim of the study was to evaluate kinetics symmetry (involved and uninvolved limb) restoration before and after ACLR, a comparison group (control group) was not included. Lastly, we did not include any self-reported questionnaires or scores for knee function, which would have added valuable information to the study.

The findings of this study showed a tendency to increase symmetry restoration in the kinetics of the involved and uninvolved limb up to twelve months after ACLR, especially in those tests in which both limbs were tested individually (gait analysis, sprint and single-leg hop tests) as opposed to those tests in which both limbs were tested simultaneously (drop vertical jump and vertical hop test). Therefore, the isolation of the involved and involved limb seems to be a critical component in the functional rehabilitation and evaluation before and after ACLR, as the uninjured contralateral extremity may tend to compensate in activities where both limbs are under stress at the same time, thus diminishing symmetry restoration.

Authors' contribution All authors were fully involved in the study and preparation of the manuscript and the material within has not been and will not be submitted for publication elsewhere.

\section{Compliance with ethical standards}

Conflict of interest The authors report no conflict of interest.

Patient consent All patients gave informed consent prior to being included in the study.

Ethical approval All procedures involving human participants were in accordance with the 1964 Helsinki Declaration and its later amendments. The study was approved by the Research Ethics Committee (or Institutional Review Board).

Funding There was no funding obtained for this study.

Open Access This article is distributed under the terms of the Creative Commons Attribution 4.0 International License (http://crea tivecommons.org/licenses/by/4.0/), which permits unrestricted use, distribution, and reproduction in any medium, provided you give appropriate credit to the original author(s) and the source, provide a link to the Creative Commons license, and indicate if changes were made.

\section{References}

1. Beynnon BD, Johnson RJ, Abate JA, Fleming BC, Nichols CE (2005) Treatment of anterior cruciate ligament injuries. Part I Am J Sports Med 33:1579-1602

2. Granan LP, Bahr R, Lie SA, Engebretsen L (2009) Timing of anterior cruciate ligament reconstructive surgery and risk of cartilage lesions and meniscal tears: a cohort study based on the Norwegian National Knee Ligament Registry. Am J Sports Med 37:955-961

3. Eitzen I, Moksnes H, Snyder-Mackler L, Risberg MA (2010) A progressive 5-week exercise therapy program leads to significant improvement in knee function early after anterior cruciate ligament injury. J Orthop Sports Phys Ther 40:705-721

4. Risberg MA, Holm I, Tjomsland O, Ljunggren E, Ekeland A (1999) Prospective study of changes in impairments and disabilities after anterior cruciate ligament reconstruction. J Orthop Sports Phys Ther 29:400-412

5. Fitzgerald GK, Lephart SM, Hwang JH, Wainner RS (2001) Hop tests as predictors of dynamic knee stability. J Orthop Sports Phys Ther 31:588-597

6. Eastlack ME, Axe MJ, Snyder-Mackler L (1999) Laxity, instability and functional outcomes after ACL injury: copers versus noncopers. Med Sci Sports Exerc 31:210-215

7. Rudolph KS, Axe MJ, Snyder-Mackler L (2000) Dynamic stability after ACL injury: who can hop? Knee Surg Sports Traumatol Arthrosc 8:262-269

8. Rudolph KS, Axe MJ, Buchanan TS, Scholz JP, Snyder-Mackler L (2001) Dynamic stability in the anterior cruciate ligament deficient knee. Knee Surg Sports Traumatol Arthrosc 9:62-71 
9. Borsa PA, Lephart SM, Irrgang JJ (1998) Comparison of performance-based and patient reported measures of function in anterior cruciate ligament-deficient individuals. J Orthop Sports Phys Ther 28:392-399

10. Daniel DM, Stone ML, Sachs R, Malcom L (1985) Instrumented measurement of anterior knee laxity in patients with acute anterior cruciate ligament disruption. Am J Sports Med 13:401-417

11. DeCarlo MS, Sell KE (1997) The effects of the number and frequency of physical therapy treatments on selected outcomes of treatment in patients with anterior cruciate ligament reconstruction. J Orthop Sports Phys Ther 26:332-339

12. Petschnig R, Baron R, Albrecht M (1998) The relationship between isokinetic quadriceps strength test and hop tests for distance and one-legged vertical jump test following anterior cruciate ligament reconstruction. J Orthop Sports Phys Ther 28:23-31

13. Fithian DC, Paxton EW, Stone ML, Luetzow WF, Csintalan RP et al (2005) Prospective trial of a treatment algorithm for the management of the anterior cruciate ligament-injured knee. Am J Sports Med 33:335-346

14. Myer GD, Schmitt LC, Brent JL, Ford KR, Foss KDB, Scherer BJ, Heidt RS, Divine JG, Hewett TE (2011) Utilization of modified NFL combine testing to identify functional deficits in athletes following ACL reconstruction. J Orthop Sports Phys Ther 41(6):377-387

15. Rohman R, Steubs JT, Tompkins M (2015) Changes in involved and uninvolved limb function during rehabilitation after anterior cruciate ligament reconstruction: implications for Limb Symmetry Index measures. Am Journal Sports Med 43(6):1391-1398

16. Logerstedt D, Lynch A, Axe M, Snyder-Mackler L (2013) Symmetry restoration and functional recovery before and after anterior cruciate ligament reconstruction. Knee Surg Sports Traumatol Arthrosc 21:859-868

17. Manal T, Snyder-Mackler L (1996) Practice guidelines for anterior cruciate ligament rehabilitation: a criterion-based rehabilitation progression. Oper Tech Orthop 6(3):190-196

18. Grindem H, Logerstedt D, Eitzen I, Moksnes H, Axe MJ, SnyderMackler L et al (2011) Single-legged hop tests as predictors of self-reported knee function in nonoperatively treated individuals with anterior cruciate ligament injury. Am J Sports Med 39:2347-2354

19. Noyes FR, Mooar PA, Matthews DS, Butler DL (1983) The symptomatic anterior cruciate-deficient knee. Part I: the longterm functional disability in athletically active individuals. J Bone Joint Surg Am 65(2):154-162
20. Lephart SM, Perrin DH, Fu FH (1992) Relationship between selected physical characteristics and functional capacity in the anterior cruciate ligament-insufficient athlete. J Orthop Sports Phys Ther 16:174-181

21. Hurd WJ, Axe MJ, Snyder-Mackler L (2008) A 10-year prospective trial of a patient management algorithm and screening examination for highly active individuals with anterior cruciate ligament injury: part 2, determinants of dynamic knee stability. Am J Sports Med 36:48-56

22. Reid A, Birmingham TB, Stratford PW, Alcock GK, Giffin JR (2007) Hop testing provides a reliable and valid outcome measure during rehabilitation after anterior cruciate ligament reconstruction. Phys Ther 87:337-349

23. Zouita Ben Moussa A, Zouita S, Dziri C, Ben Salah BZ (2009) Single-leg assessment of postural stability and knee functional outcome two years after anterior cruciate ligament reconstruction. Ann Phys Rehab Med 52:475-484

24. Noyes FR, Barber SD, Mangine RE (1991) Abnormal lower limb symmetry determined by function hop tests after anterior cruciate ligament rupture. Am J Sports Med 19:513-518

25. Kvist J (2004) Rehabilitation following anterior cruciate ligament injury: current recommendations for sports participation. Sports Med 34:269-280

26. Gustavsson A, Neeter C, Thomeé P, Silbernagel KG, Augustsson J, Thomeé R et al (2006) A test battery for evaluating hop performance in patients with an ACL injury and patients who have undergone ACL reconstruction. Knee Surg Sports Traumatol Arthrosc 14:778-788

27. Paterno MV, Schmidt LC, Ford KR et al (2010) Biomechanical measures during landing and postural stability predict second anterior cruciate ligament injury after anterior cruciate ligament reconstruction and return to sport. Am J Sports Med 38(10): 1968-1978

28. Ardern CL, Webster KE, Taylor NF, Feller JA (2011) Return to the preinjury level of competitive sport after anterior cruciate ligament reconstruction surgery: two thirds of patients have not returned by 12 months after surgery. Am J Sports Med 39(3):538-543

29. Adams D, Logerstedt DS, Hunter-Giordiano A et al (2012) Current concepts for anterior cruciate ligament reconstruction: a criterion-based rehabilitation progression. J Orthop Sports Phys Ther 42(7):601-614

30. Kramer JF, Nusca D, Fowler P, Webster-Bogaert S (1992) Testretest reliability of the one-leg hop test following ACL reconstruction. Clin J Sport Med 2(4):240-243 